\title{
Improving the Appropriateness of Antipsychotic Prescribing for Behavioral and Psychological Symptoms of Dementia (BPSD): A Pilot Study of the Psychotropic Use Monitoring (PUM) Program
}

\author{
Kai Zhen Yap1, Ee Heok Kua², Sui Yung Chan', Joyce Yu-Chia Lee ${ }^{{ }^{*}}$ \\ ${ }^{1}$ Department of Pharmacy, National University of Singapore, Singapore, Republic of Singapore \\ ${ }^{2}$ Department of Psychological Medicine, National University Health System, Singapore, Republic of Singapore \\ Email: ${ }^{*}$ phalycj@nus.edu.sg
}

Received 22 March 2014; revised 15 April 2014; accepted 23 April 2014

Copyright (C) 2014 by authors and Scientific Research Publishing Inc.

This work is licensed under the Creative Commons Attribution International License (CC BY). http://creativecommons.org/licenses/by/4.0/

\section{Open Access}

\section{Abstract}

In nursing homes, antipsychotic prescribing decisions (APDs) for managing behavioral and psychological symptoms of dementia (BPSD) depend on the nursing staff's feedback. Inappropriate APDs can result in the lack of timeliness, objectivity and important clinical information when nursing staff's feedback on residents' behavior and pharmacotherapy outcomes. Currently, there are no reported interventions for improving psychiatrists' APDs through nursing staff's monitoring and feedback processes. This one-group pre-and-post pilot study aimed to evaluate the feasibility and impact of implementing a newly-developed Psychotropic Use Monitoring (PUM) program for improving the appropriateness of APDs in a 50-bed dementia ward of a nursing home. The PUM intervention involved 16 pharmacist-trained nursing staff, who monitored and reported residents' BPSD changes and psychotropic side effects for 24 weeks, while carrying out their routine care duties. A face-to-face interview was then administered to determine the nursing staff's perceptions of PUM. Data of 51 residents were collected from hardcopy individual patient records to evaluate the changes in APDs and the number of resident falls before and after implementing PUM. The nursing staff reported increases in their knowledge, awareness, confidence, and actual frequency of monitoring for side effects, as well as their ability in differentiating and managing BPSD ( $p$ < 0.05). After PUM, there was a significant increase in the number of APDs due to side effect-related reasons $(4$ versus 16$)(p<0.031)$. Although not significant, the number of APDs with no documented reasons ( 5 versus 9 ) and the number of resident falls ( 7 versus 15 ) appeared to be lesser after PUM. This study demonstrated the nursing staff's positive participation in PUM inter-

\footnotetext{
${ }^{*}$ Corresponding author.
} 
vention, specifically in monitoring and feedback of side effects. Furthermore, a potential exists for PUM to encourage more judicious APDs, which may be useful in settings with heavy patient load, limited human resources and dependence on foreign nursing staff from differing cultural backgrounds.

\section{Keywords}

\section{Antipsychotics, Appropriate Prescribing, Behavioral and Psychological Symptoms of Dementia, Nursing Home, Falls}

\section{Introduction}

Behavioral and Psychological Symptoms of Dementia (BPSD) is present in up to 97\% of persons with dementia over a five-year period [1], and often result in patient distress, caregiver stress, increased cost of care, nursing home admissions as well as a corresponding higher prevalence of antipsychotic use in the nursing homes compared to the social care setting [2]. Despite limited long-term benefits, numerous side effects, and associated higher risks of stroke and death [3], the use of antipsychotics for managing symptoms of severe agitation, aggression and psychosis in the nursing homes will likely continue, especially when these symptoms pose significant threats to the safety of the resident and those around him/her [4] [5]. Hence, ensuring the appropriateness of antipsychotic prescribing decisions (APDs) is of paramount importance [6].

APDs to manage BPSD in the nursing homes depend primarily on nursing staff's feedback about residents' behavior and pharmacotherapy outcomes, especially from the nursing aides (NA) and healthcare attendants (HA), who form up to two-thirds of the staff strength [7] and have the closest daily contact with the residents [8]. However, the lack of formal healthcare education among the NA and HA [9], inadequate monitoring processes in the nursing homes [10], caregiving stress due to low staff-to-resident ratio [6] and nurse-resident miscommunication due to cultural and language barriers [8] [11] may result in feedback that is delayed, subjective or lacking in important clinical information [12]. Such inadequate monitoring and feedback processes thus contribute to inappropriate APDs, specifically "over-prescribing" of antipsychotics without a clear indication or when they are no longer indicated, "mis-prescribing" of antipsychotics due to mis-identification of underlying target symptoms to be treated and "under-prescribing" of antipsychotics for residents who may benefit from short-term use of antipsychotics [13].

In the past decade, many interventions to improve APDs in the nursing homes were reported [14]-[23]. To our knowledge, there were no interventions targeted at improving APDs for managing BPSD through a structured nursing staff's monitoring and feedback process. In addition, all but two of these 10 reported studies did not evaluate the interventions' impact on adverse outcomes such as falls, possibly associated with antipsychotic use and/or severe BPSD [24] [25].

Hence, the Psychotropic Use Monitoring (PUM) program was developed by the authors to improve psychiatrists' APDs for alleviating BPSD while minimizing adverse drug outcomes, through the structured intervention of timely and objective monitoring and feedback by the nursing staff of residents' target symptoms, treatment responses and side effects of psychotropics, especially antipsychotics. The objective of this pilot study was to evaluate the PUM program's impact on the nursing staff involved, APDs and number of resident falls.

\section{Methods}

\subsection{Study Design, Setting and Participants}

This was a one-group pre- and post-test pilot study. After obtaining approval from the authors' university institutional review board and the nursing home involved, the PUM program was implemented in the 50-bed dementia ward of the nursing home. The nursing home was attended by a psychiatrist of a nearby hospital every two weeks. After receiving the PUM-related training, the 16 nursing staff at the ward carried out the PUM intervention for a period of 24 weeks from February to August 2010. Specifically, these nursing staff were foreign employees from Myanmar $(n=10)$ and the Philippines $(n=6)$, among whom were three certified nurses, six NA 
and seven HA, with a mean (SD) of 0.4 (0.3), 1.3 (0.8), and 1.3 (1.7) years' experience working in a dementia ward setting respectively. After the 24-week intervention period, these nursing staff participated in a survey on their perception of PUM, while the relevant data of the 51 residents who were present during 24-weeks before and after implementing PUM were used for evaluating PUM's impact on the APDs and number of resident falls.

\subsection{Description of PUM-Related Training}

The PUM-related training was provided to the participating nursing staff prior to the start of the 24-week PUM intervention. The training included one introductory (before implementing PUM) and three review sessions (at Weeks 4, 8 and 12 after implementing PUM), for the purpose of (1) communicating the significance of nursing staff's role in supporting the appropriate use of antipsychotics in the management of BPSD (2) demonstrating the use of the PUM form and the clinical observation/evaluation skills required for the intervention, and (3) providing continual review of the intervention. All the sessions were conducted at the premises of the nursing home by the pharmacist. In order to accommodate the shift duty and facilitate the attendance of all participating nursing staff, each session was repeated within a week after the originally scheduled date.

\subsection{Description of PUM Intervention}

The PUM intervention required the nursing staff to observe the residents for BPSD changes and psychotropic side effects while they carried out their routine care duties, according to the intervention protocol illustrated in Figure 1. The nursing staff documented their observations on the specially-developed PUM form (Steps 2 and 6 of protocol), which were then reviewed by the pharmacist and nurse in-charge of the ward and escalated to the regular visiting psychiatrist for further action, during his fortnightly consultations at the nursing home (Steps 3, 4, 7 and 8 of protocol). As the PUM intervention was based on observations, the nursing staff were not required to spend extra time in conducting additional interviews/physical assessments on the residents. To ensure the compliance of the nursing staff to the scheduled monitoring of the residents with prescribed changes in their psychotropic medications, the pharmacist (Step 5 of protocol) drafted and regularly updated a log for communicating the dates when monitoring should take place, along with notes on the medication use outcomes that will likely be observed by the nursing staff.

\subsection{Description of the PUM Form}

The PUM form contained three sections, namely, (1) an Assessment for Psychotropic Prescriptions (APP) scale to document residents' behavioral changes according to the different BPSD "types" for better identification of target symptoms and treatment responses, (2) a section to document resident's current psychotropics for discerning and attributing the observations as treatment and/or side effects of the respective pharmacological agents which may also be prescribed with antipsychotics for managing BPSD, and (3) a checklist for documenting postulated psychotropic side effects. An example of the PUM form is shown in the Appendix.

The BPSD “types” in the APP scale included sleep and appetite disturbances, anxiety, agitation/irritability, aggression, depression, elation, disinhibition, delusion, and hallucination. To ensure the objectivity and clinical relevance of the nursing staff's feedback on residents' behavioral changes, explicit behavior descriptors were developed from the expert opinion of the clinical investigators to serve as anchors for rating the severity of the respective BPSD “types”. Prior to the study, the APP scale was validated and found to have satisfactory inter-rater reliability among the users and concurrent validity with the Neuropsychiatric Inventory [26]. Details of the explicit behavior descriptors and validation tests are obtainable from the corresponding author.

The checklist of psychotropic side effects was compiled from drug references [27]-[29]. To increase the user-friendliness of the checklist and encourage reporting by the nursing staff, only SEs that are common and easily observable were listed explicitly with checkboxes, while space is provided under the checkbox listed as "others" for the nursing staff to enter their observations freely, which may include descriptions of drug-induced movement disorders or findings from routine resident assessments such as orthostatic hypotension.

\subsection{Outcome Measures and Data Collection}

The first outcome was the nursing staff's self-reported changes in behavior, knowledge, attitudes, ability, and 


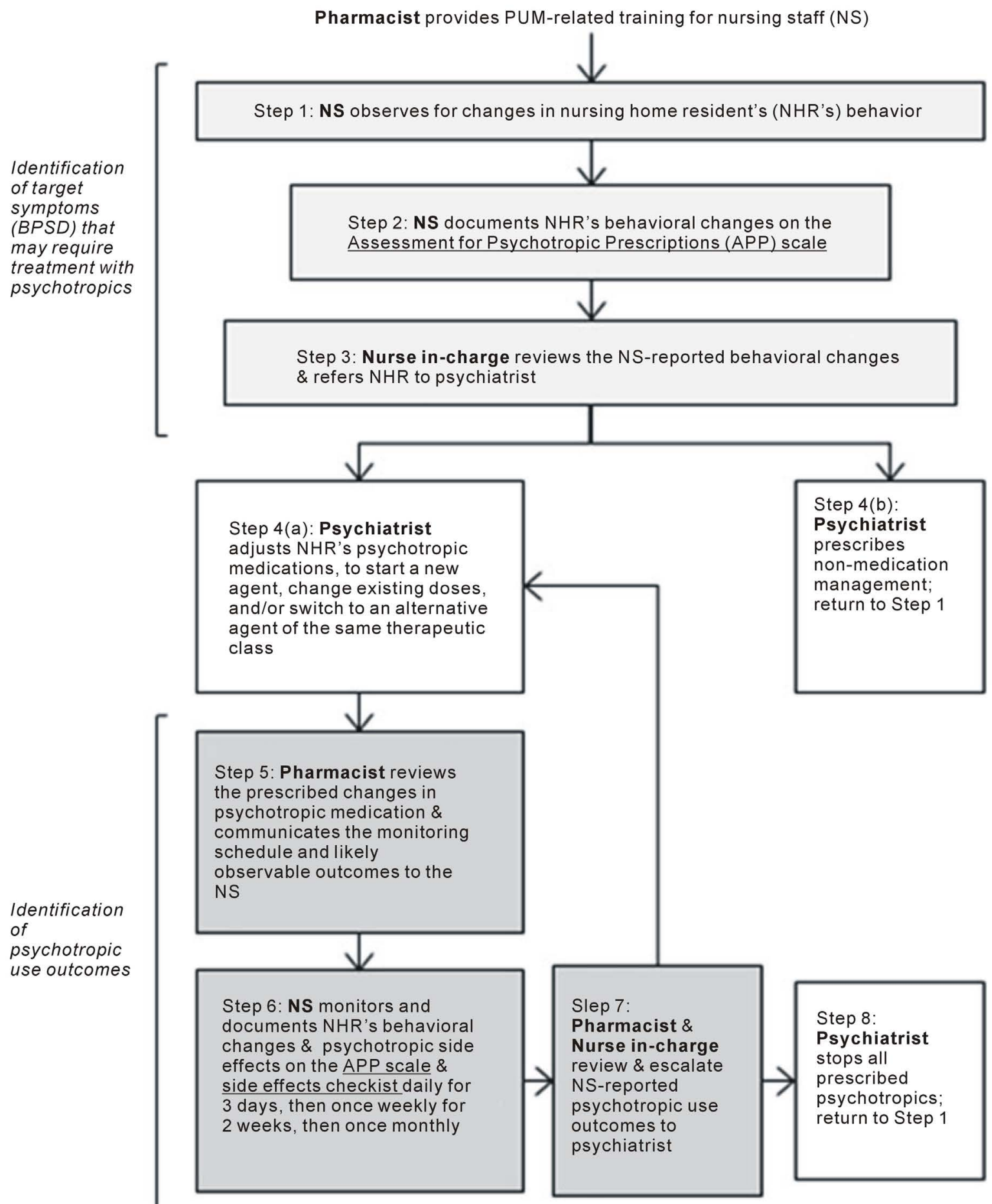

Figure 1. Psychotropic Use Monitoring (PUM) Intervention Protocol.

caregiving stress over 24-week periods before and after implementing PUM (Table 1). These were captured using a structured interviewer-administered survey, conducted by the author in English for each nursing staff individually during the week after the 24-week intervention period, in a private area of the nursing home, at a time that was convenient for the respondents. Before the start of the survey, the anonymity of the respondents was assured so as to minimize potential response bias. The nursing staff were also encouraged to think aloud during 
Table 1. Perception and Behavioral Changes of Nursing Staff $(n=16)$.

\begin{tabular}{|c|c|c|c|c|c|}
\hline \multirow{2}{*}{ Description of Survey Item } & \multicolumn{2}{|c|}{ Median Rating (IQ Range) ${ }^{\mathrm{a}}$} & \multirow{2}{*}{ P-value ${ }^{b}$} & \multirow{2}{*}{$\begin{array}{l}\text { Number of NS } \\
\text { with Positive } \\
\text { Change }^{c}\end{array}$} & \multirow{2}{*}{ P-value $^{d}$} \\
\hline & Before PUM & After PUM & & & \\
\hline \multicolumn{6}{|l|}{ Changes in Behavior } \\
\hline frequency in managing BPSD & $1.5(0.3-2.0)$ & $1.5(0.3-2.0)$ & 0.004 & 10 & 0.454 \\
\hline frequency in monitoring SE & $1.5(0-2.0)$ & $3.0(2.0-3.0)$ & 0.001 & 14 & 0.004 \\
\hline \multicolumn{6}{|l|}{ Changes in Knowledge } \\
\hline on BPSD & $1.0(0.3-2.0)$ & $3.0(2.0-3.0)$ & 0.002 & 12 & 0.077 \\
\hline on SE & $1.0(1.0-2.0)$ & $3.0(3.0-3.0)$ & $<0.001$ & 15 & 0.001 \\
\hline \multicolumn{6}{|l|}{ Changes in Attitudes towards SE } \\
\hline awareness to monitor & $1.0(1.0-1.8)$ & $3.0(3.0-4.0)$ & $<0.001$ & 16 & $<0.001$ \\
\hline confidence to correctly identify & $1.0(1.0-2.0)$ & $3.0(3.0-4.0)$ & 0.001 & 14 & 0.004 \\
\hline \multicolumn{6}{|l|}{ Changes in Ability } \\
\hline manage BPSD well & $1.5(1.0-2.0)$ & $3.0(2.0-3.0)$ & 0.001 & 14 & 0.004 \\
\hline differentiate BPSD types \& severity & $1.0(0-2.0)$ & $3.0(2.0-3.0)$ & 0.001 & 15 & 0.001 \\
\hline recognize SE & $1.5(1.0-2.0)$ & $3.0(3.0-3.0)$ & 0.001 & 14 & 0.004 \\
\hline \multicolumn{6}{|c|}{ Changes in Perceived Stress (during caregiving) } \\
\hline feel stressful when managing BPSD & $1.0(1.0-2.8)$ & $1.0(1.0-2.8)$ & 0.971 & 7 & 0.804 \\
\hline
\end{tabular}

BPSD = behavioral and psychological symptoms of dementia, IQ = inter-quartile, NS = nursing staff, PUM = Psychotropic Use Monitoring, SD = standard deviation, SE = side effect; a. Ratings were made on a 5-point scale, with increasing intensity from " 0 " to " 4 ". These ratings corresponded to "not at all”, "rarely"/”a little bit”, "some of the time”/moderately", "most of the time"/"quite a bit”, and "always"/”extremely". b. Wilcoxon Signed Ranks test (2-sided); c. Positive change was defined as a difference (After-Before) of $<0$ in rating with regards to perceived stress, and a difference (After-Before) of $>0$ in rating with regards to other items. d. Binomial test (2-sided).

the survey, while explanations of the survey questions were provided when deemed necessary, to verify the survey responses and minimize misunderstanding.

The second outcome was the changes in APDs over the 24-week intervention period compared with that over the 24-week period prior to PUM implementation. Specifically, the APDs made by the nursing home's visiting psychiatrist over 24-week periods before and after implementing PUM were transcribed from the original hardcopy of the residents' medical prescribing notes, after the study period. These APDs were categorized and compared according to the reasons ("BPSD-related", "side effect-related" and "no documented reason") and types ("start new", "increase dose", "decrease dose", and "discontinue”).

The third outcome was the change in the number of resident falls over 24 weeks before and after implementing PUM. The number of resident falls was obtained directly from the original incident reports that were submitted by the nursing staff to the nursing home administrator's office independently from the study.

\subsection{Statistical Analysis}

All statistical analyses were performed on IBM SPSS Statistics for Windows, Version 19.0 (IBM Corp., Armonk, NY). A minimum sample size of 20 to 25 nursing staff survey responses and resident data sets was targeted in this study as recommended for evaluating the efficacy of interventions in a single group [30]. As the estimated sample sizes were small, nonparametric statistical tests were used. Specifically, the Wilcoxon signed rank test and the binomial test (using test proportion of 0.5) were used to evaluate the nursing staff's responses in the survey. Taking each APDs as independent units of analysis, the chi-square test (with Bonferroni adjustments) was used to evaluate the differences in the APDs due to "BPSD-related" reasons, "side effect-related" reasons, and "no documented reasons" made before and after implementing PUM. Lastly, the McNemar test was used to evaluate difference in the number of falls reported before and after PUM. Estimates with P value < 0.05 were considered to be statistically significant. In addition, the demographic characteristics of the residents (from whom APD and falls data were obtained) were reported descriptively. The mean duration and the mean average daily dose of antipsychotics used by the included residents during each study period were also reported descriptively as resident-days and chlorpromazine equivalent doses, calculated based on published literature [31] [32] and drug reference [29] respectively. 


\section{Results}

\subsection{Self-Reported Changes in Nursing Staff Perception of PUM and Behavior}

All 16 nursing staff involved in the PUM program participated in the survey. Increases in their frequency of monitoring side effects, knowledge on side effects, awareness and confidence to monitor for and identify side effects, and their ability in managing and identifying BPSD and side effects during the intervention period were reported by 14 or more $(\mathrm{p}<0.05)$ of the 16 nursing staff surveyed (Table 1 ).

\subsection{Resident Characteristics, Changes in Antipsychotic Prescribing Decisions (APDs) and Number of Resident Falls}

The data of 51 residents whose mean (SD) age was 79.8 (9.5) years and duration of residence was 163.0 (21.9) and 150.8 (42.0) days before and after implementing PUM respectively were included in the analyses. More than half of them were male (54.9\%), Chinese (74.5\%), and used five or more regular medications (60.8\%). Vascular dementia, other/mixed dementia, Alzheimer's dementia, and unspecified dementia were diagnosed among $37.2 \%, 31.4 \%, 29.4 \%$, and $2 \%$ of these residents. The mean (SD) duration of antipsychotic use was 0.39 (0.47) and $0.48(0.48)$ resident-days before and after implementing PUM respectively. While antipsychotics were prescribed for 23 and 27 residents before and after PUM implementation, the mean average daily dose used by these residents was lower ( $67.3 \pm 51.8 \mathrm{mg} /$ day versus $70.2 \pm 63.2 \mathrm{mg} /$ day) after implementing PUM.

After implementing PUM, the total number of APDs was higher (47 versus 34). Specifically, the APDs due to "side effect-related" reasons (16 versus 4) were found to be significantly higher, as shown in Table 2 ( $\mathrm{p}<$ 0.031). Although the proportion of APDs with "no reasons documented" was lower after implementing PUM (5 versus 9), this was not statistically significant. From Table 2, while the number of APDs to "start new" due to "BPSD-related" reasons did not change $(n=9)$, the number of APDs to "increase dose" due to "BPSD-related" reasons appeared to be higher after implementing PUM (17 versus 6). Interestingly, APDs to switch antipsychotic agents ( $n=5$, where "start new" occurred with corresponding "discontinue") due to "side effect-related" reasons were observed only after implementing PUM. Although fewer resident falls were reported after implementing PUM (7 versus 15), this was not statistically significant.

\section{Discussion}

\subsection{Unique Characteristics of the PUM Intervention}

Published interventions that aimed to improve the appropriateness of antipsychotic use in the nursing homes in

Table 2. Number of APDs by Reasons Before $(n=34)$ and After $(n=47)$ Implementing PUM.

\begin{tabular}{|c|c|c|c|}
\hline \multirow{2}{*}{ APDs } & \multicolumn{2}{|c|}{ Number of APDs } & \multirow{2}{*}{ P-value } \\
\hline & Before PUM $(n=34)$ & After PUM (n = 47) & \\
\hline No reasons documented & 9 & 5 & 0.031 \\
\hline "Start new" & 2 & 1 & \\
\hline "Increase dose" & 1 & 2 & \\
\hline “Discontinue” & 6 & 2 & \\
\hline BPSD-related & 21 & 26 & \\
\hline "Start new" & 9 & 9 & \\
\hline "Increase dose" & 6 & 17 & \\
\hline "Decrease dose" & 2 & 0 & \\
\hline “Discontinue” & 4 & 0 & \\
\hline SE-related ${ }^{b}$ & 4 & 16 & \\
\hline "Start new" & 0 & 5 & \\
\hline "Decrease dose" & 2 & 5 & \\
\hline “Discontinue" & 2 & 6 & \\
\hline
\end{tabular}

APD = antipsychotic prescribing decision, BPSD = behavioral and psychological symptoms of dementia, PUM = Psychotropic Use Monitoring, SE = side effect; a. Chi-square test was used for comparing the proportions of APDs (by reasons) before and after implementing PUM. b. Using the Bonferroni adjustment, the significant p-value of 0.031 was attributed to the difference between the proportions of APDs due to SE-related reasons recorded before and after implementing PUM.” 
cluded audits [14] [22] [23], medication reviews [21], education [15] [17] [20], multidisciplinary case-conferencing [19], early screening [16] and non-pharmacological management [18]. PUM is unique among these interventions as it emphasizes on the monitoring process, leveraging on the capabilities and roles of the pharmacist as the educator and advocator for appropriate medication use [33] and the nursing staff as the keen first-hand observer [34], to support the appropriate APDs by the visiting psychiatrist. While the use of the validated APP scale in the PUM form ensured the objectivity and clinical relevance of the nursing staff's monitoring and feedback, the observation-based PUM intervention enabled the nursing staff to monitor residents efficiently and overcome potential language barriers between the majority of non-English-speaking residents and nursing staff (or English-speaking residents and non-English-speaking nursing staff) when assessing BPSD changes and side effects. Thus, PUM may be helpful in nursing homes of many countries, including the US, which face challenges of increasingly heavy patient load, limited human resources [11], high dependence on immigrant and non-immigrant nursing staff from differing cultural backgrounds and diverse resident demographics in terms of race and ethnicity [35].

\subsection{Feasibility and Impact of Implementing PUM}

The survey results suggested that the nursing staff, comprising mainly of NAs and HAs, were motivated and engaged to carry out PUM, especially in monitoring of side effects. This observation was corroborated by the resulting increase in the proportion of "side effect-related" APDs made after implementing PUM. In addition, the visiting psychiatrist also feedback that the nursing staff's frequency of reporting psychotropic side effects had "increased moderately", while their frequency of correctly identifying side effects had "increased a little bit" after PUM was implemented.

After implementing PUM, APDs appeared to be more accountable, with fewer decisions having "no documented reasons". Furthermore, the lower average mean daily antipsychotic dose used after PUM implementation despite the increased number of "BPSD-related" antipsychotic dose increases suggested that APDs were more cautious, with more frequent but smaller dose adjustments, in line with the guidelines which recommend for appropriate APDs to "start low and go slow" [36].

The reduced number of falls despite the increased duration and prevalence of antipsychotic use could be due to the increased APDs to minimize the nursing staff-reported fall-inducing side effects of drowsiness and extrapyramidal symptoms [24], while continuing pharmacological management of severe agitation, aggression and psychosis to prevent risky behaviors or use of restraints, which may also contribute to falls [25]. However, due to the multifactorial nature of falls, this study was not able to adequately establish the direct effect of the observed increase in APDs to reduce antipsychotic side effects on the observed reduction in falls. On the other hand, the PUM intervention's potential in lowering the number of falls may be attributed to the nursing staff's increased safety awareness and vigilance to prevent resident falls. This was alluded by six nursing staff during the survey, who reported feeling more "stressful" and responsible towards the residents as they become more aware of the adverse repercussions of psychotropic side effects (such as falls) and the importance to monitor and manage them.

\subsection{Study Limitations}

The major limitation of this pilot study was the small resident sample size, which possibly resulted in the lack of statistical significance and generalizability of the findings. Although the use of a face-to-face survey could have led to biased responses from the nursing staff, these were minimized by assuring respondent's anonymity and privacy, and verifying the quantitative responses during the interview. Nonetheless, a cluster randomized controlled study would be needed to further assess the impact of PUM on the APDs and resident outcomes such as falls and BPSD changes in other nursing home settings, as well as the trends of antipsychotic use in comparison with other psychotropics such as benzodiazepines, antidepressants and mood stabilizers that may be prescribed for managing BPSD [37].

\section{Conclusion}

This pilot study demonstrated the PUM program's positive influences on the attitudes of the nursing staff towards stepping up on the objective and timely monitoring and feedback of residents' behavior, and especially 
that of psychotropic side effects. This in turn encouraged more cautious APDs by the visiting psychiatrist for the management of BPSD, especially in minimizing the medication's side effects. Hence, the efficient PUM intervention may be useful to address inappropriate APDs among the elderly with dementia, especially in settings with heavy patient load, limited human resources and dependence on foreign nursing staff from differing cultural backgrounds. As such, this pilot study provides a basis for larger cluster randomized controlled studies to be carried out, to ascertain the impact of the PUM program on reducing inappropriate APDs and resident falls in other nursing homes and practice settings.

\section{Acknowledgements}

The authors acknowledge Dr. Feng Lei and the clinical team of the participating nursing home for their assistance during the validation of the PUM form, and Ms. Melanie Siaw for her assistance in data collection.

\section{Conflict of Interest}

The authors declare no conflict of interest.

\section{Financial Disclosure}

This study was supported by the National University of Singapore (NUS) Virtual Institute for the Study of Ageing (VISA) Grant and the Singapore Ministry of Education (MOE) Research Scholarship, which supported the postgraduate studies of the first author during which this study was conducted.

\section{Author Contributions}

All authors contributed to the concept, design, coordination, data collection, analysis and interpretation of this study.

\section{References}

[1] Steinberg, M., Shao, H., Zandi, P., Lyketsos, C.G., Welsh-Bohmer, K.A., Norton, M.C., et al. (2008) Point and 5-Year Period Prevalence of Neuropsychiatric Symptoms in Dementia: The Cache County Study. International Journal of Geriatric Psychiatry, 23, 170-177. http://dx.doi.org/10.1002/gps.1858

[2] Shah, A., Carey, I. M., Harris, T., DeWilde, S. and Cook, D.G. (2011) Antipsychotic Prescribing to Older People Living in Care Homes and the Community in England and Wales. International Journal of Geriatric Psychiatry, 26, 423434. http://dx.doi.org/10.1002/gps.2557

[3] Schneider, L.S., Dagerman, K.S. and Insel, P. (2005) Risk of Death with Atypical Antipsychotic Drug Treatment for Dementia. Meta-Analysis of Randomized Placebo-Controlled Trials. JAMA, 294, 1934-1943. http://dx.doi.org/10.1001/jama.294.15.1934

[4] Schultz, S.K. (2008) Atypical Antipsychotic Medications in Alzheimer's Disease: Effectiveness versus Expectations. American Journal of Psychiatry, 165, 787-789. http://dx.doi.org/10.1176/appi.ajp.2008.08040517

[5] Declercq, T., Petrovic, M., Vander Stichele, R., De Sutter, A.I.M., van Driel, M.L. and Christiaens, T. (2013) Withdrawal versus Continuation of Chronic Antipsychotic Drugs for Behavioural and Psychological Symptoms in Older People with Dementia. Cochrane Database of Systematic Review, Article ID: CD007726.

[6] Crystal, S., Olfson, M., Huang, C., Pincus, H. and Gerhard, T. (2009) Broadened Use of Atypical Antipsychotics: Safety, Effectiveness, and Policy Challenges. Health Affairs, 28, w770-781. http://dx.doi.org/10.1377/hlthaff.28.5.w770

[7] Leung, T. and Menon, S. (2011) Who Will Look after Our Elderly? April 29, 2012. http://www.siiaonline.org/?q=programmes/commentary/who-will-look-after-our-elderly

[8] Tellis-Nayak, V. and Tellis-Nayak, M. (1989) Quality of Care and the Burden of Two Cultures: When the World of the Nurse's Aide Enters the World of the Nursing Home. The Gerontologist, 29, 307-313. http://dx.doi.org/10.1093/geront/29.3.307

[9] Lindquist, L.A., Jain, N., Tam, K., Martin, G.J. and Baker, D.W. (2009) Inadequate Health Literacy among Paid Caregivers of Seniors. Journal of General Internal Medicine, 26, 474-479. http://dx.doi.org/10.1007/s11606-010-1596-2

[10] Barher, N.D., Alldred, D.P., Raynor, D.K., Dickinson, R., Garfield, S., Jessen, B., et al. (2009) Care homes’ Use of Medicines Study: Prevalence, Causes and Potential Harm of Medication Errors in Care Homes for Older People. Qual- 
ity \& Safety in Health Care, 18, 341-346. http://dx.doi.org/10.1136/qshc.2009.034231

[11] Khatutsky, G., Wiener, J.M. and Anderson, W.L. (2010) Immigrant and Non-Immigrant Certified Nursing Assistants in Nursing Homes: How Do They Differ? Journal of Aging \& Social Policy, 22, 267-287. http://dx.doi.org/10.1080/08959420.2010.485526

[12] Mamun, K., Goh-Tan, C.Y.E. and Ng, L.L. (2003) Prescribing Psychoactive Medications in Nursing Homes: Current Practice in Singapore. Singapore Medical Journal, 44, 625-629.

[13] Buetow, S.A., Sibbald, B., Cantrill, J.A. and Halliwell, S. (1997) Appropriateness in Health Care: Application to Prescribing. Social Science \& Medicine, 45, 261-271. http://dx.doi.org/10.1016/S0277-9536(96)00342-5

[14] Castle, N.G. (2003) Providing Outcomes Information to Nursing Homes: Can It Improve Quality of Care? The Gerontologist, 43, 483-492. http://dx.doi.org/10.1093/geront/43.4.483

[15] Hagen, B.F., Armstrong-Esther, C., Quail, P., Williams, R.J., Norton, P., Le Navenec, C.-L., et al. (2005) Neuroleptic and Benzodiazepine Use in Long-Term Care in Urban and Rural Alberta: Characteristics and Results of an Education Intervention to Ensure Appropriate Use. International Psychogeriatrics, 17, 631-652. http://dx.doi.org/10.1017/S1041610205002188

[16] Kotynia-English, R., McGowan, H. and Almeida, O. (2005) A Randomized Trial of Early Psychiatric Intervention in Residential Care: Impact on Health Outcomes. International Psychogeriatrics, 17, 475-485. http://dx.doi.org/10.1017/S1041610205001572

[17] Fossey, J., Ballard, C., Juszczak, E., James, I., Alder, N., Jacoby, R., et al. (2006) Effect of Enhanced Psychosocial Care on Antipsychotic Use in Nursing Home Residents with Severe Dementia: Cluster Randomised Trial. British Medical Journal, 332, 756-761. http://dx.doi.org/10.1136/bmj.38782.575868.7C

[18] Putman, L. and Wang, J.T. (2007) The Closing Group: Therapeutic Recreation for Nursing Home Residents with Dementia and Accompanying Agitation and/or Anxiety. American Journal of Alzheimer's Disease \& Other Dementias, 22, 167-175. http://dx.doi.org/10.1177/1533317507300514

[19] Dahl, L.J., Wright, R.J., Xiao, A., Keeven, A. and Carr, D.B. (2008) Quality Improvement in Long Term Care: The Psychotropic Assessment Tool (PAT). Journal of the American Medical Directors Association, 9, 676-683. http://dx.doi.org/10.1016/j.jamda.2008.07.002

[20] Monette, J., Champoux, N., Monette, M., Fournier, L., Wolfson, C., Galbaud du Fort, G., Sourial, N., Le Cruguel, J.P. and Gore, B. (2008) Effect of an Interdisciplinary Educational Program on Antipsychotic Prescribing among Nursing Home Residents with Dementia. International Journal of Geriatric Psychiatry, 23, 574-579. http://dx.doi.org/10.1002/gps.1934

[21] Patterson, S.M., Hughes, C.M., Crealey, G., Cardwell, C. and Lapane, K.L. (2010) An Evaluation of an Adapted U.S. Model of Pharmaceutical Care to Improve Psychoactive Prescribing for Nursing Home Residents in Northern Ireland (Fleetwood Northern Ireland Study). Journal of the American Geriatrics Society, 58, 44-53. http://dx.doi.org/10.1111/j.1532-5415.2009.02617.x

[22] Westbury, J., Jackson, S., Gee, P. and Peterson, G. (2010) An Effective Approach to Decrease Antipsychotic and Benzodiazepine Use in Nursing Homes: The RedUSe Project. International Psychogeriatrics, 22, 26-36. http://dx.doi.org/10.1017/S1041610209991128

[23] Westbury, J., Tichelaar, L., Peterson, G., Gee, P. and Jackson, S. (2011) A 12-Month Follow-Up Study of "RedUSe”: A Trial Aimed at Reducing Antipsychotic and Benzodiazepine Use in Nursing Homes. International Psychogeriatrics, 23, 1260-1269. http://dx.doi.org/10.1017/S1041610211000421

[24] Leipzig, R.M., Cumming, R.G. and Tinetti, M.E. (1999) Drugs and Falls in Older People: A Systematic Review and Meta-Analysis: I. Psychotropic Drugs. Journal of the American Geriatrics Society, 47, 30-39.

[25] Sylliaas, H., Selbaek, G. and Bergland, A. (2012) Do Behavioral Disturbances Predict Falls among Nursing Home Residents? Aging Clinical and Experimental Research, 24, 251-256.

[26] Cummings, J.L. (1997) The Neuropsychiatric Inventory: Assessing Psychopathology in Dementia Patients. Neurology, 48, S10-S16. http://dx.doi.org/10.1212/WNL.48.5_Suppl_6.10S

[27] (2013) DRUGDEX ${ }^{\circledR}$ System (Micromedex 2.0). http://www.micromedexsolutions.com/home/dispatch

[28] Semla, T.P., Beizer, J.L. and Higbee, M.D. (2008) Geriatric Dosage Handbook. 14th Edition, Lexi-Comp, Inc., Hudson.

[29] Joint Formulary Committee of the British Medical Association and the Pharmaceutical Society of Great Britain (2011) British National Formulary (BNF 61). Pharmaceutical Press, London.

[30] Hertzog, M. (2008) Considerations in Determining Sample Size for Pilot Studies. Research in Nursing \& Health, 31, 180-191. http://dx.doi.org/10.1002/nur.20247

[31] Kane, J.M., Aguglia, E., Altamura, A.C., Gutierrez, J.L.A., Brunello, N., Fleischhacker, W.W., et al. (1998) Guidelines for Depot Antipsychotic Treatment in Schizophrenia. European Neuropsychopharmacology, 8, 55-66. 
http://dx.doi.org/10.1016/S0924-977X(97)00045-X

[32] Woods, S.W. (2003) Chlorpromazine Equivalent Doses for the Newer Atypical Antipsychotics. Journal of Clinical Psychiatry, 64, 663-667. http://dx.doi.org/10.4088/JCP.v64n0607

[33] Stuart, A. (2002) The State of the World's Pharmacy: A Portrait of the Pharmacy Profession. Journal of Interprofessional Care, 16, 391-404. http://dx.doi.org/10.1080/1356182021000008337

[34] Jordan, S., Tunnicliffe, C. and Sykes, A. (2002) Minimizing Side-Effects: The Clinical Impact of Nurse-Administered 'Side-Effect' Checklists. Journal of Advanced Nursing, 37, 155-165. http://dx.doi.org/10.1046/j.1365-2648.2002.02064.x

[35] Feng, Z., Fennell, M.L., Typer, D.A., Clark, M. and Mor, V. (2011) Growth of Racial and Ethnic Minorities in US Nursing Homes Driven by Demographics and Possible Disparities in Options. Health Affairs, 30, 1358-1365. http://dx.doi.org/10.1377/hlthaff.2011.0126

[36] British Columbia Ministry of Health (2012) Best Practice Guideline for Accommodating and Managing Behavioural and Psychological Symptoms of Dementia in Residential Care. 31 October 2013.

http://www.health.gov.bc.ca/library/publications/year/2012/bpsd-guideline.pdf

[37] Lester, P., Kohen, I., Stefanacci, R.G. and Feuerman, M. (2011) Antipsychotic Drug Use since the FDA Black Box Warning: Survey of Nursing Home Policies. Journal of the American Medical Directors Association, 12, 573-577. http://dx.doi.org/10.1016/j.jamda.2010.04.005

\section{Appendix-Psychotropic Use Monitoring (PUM) Form}

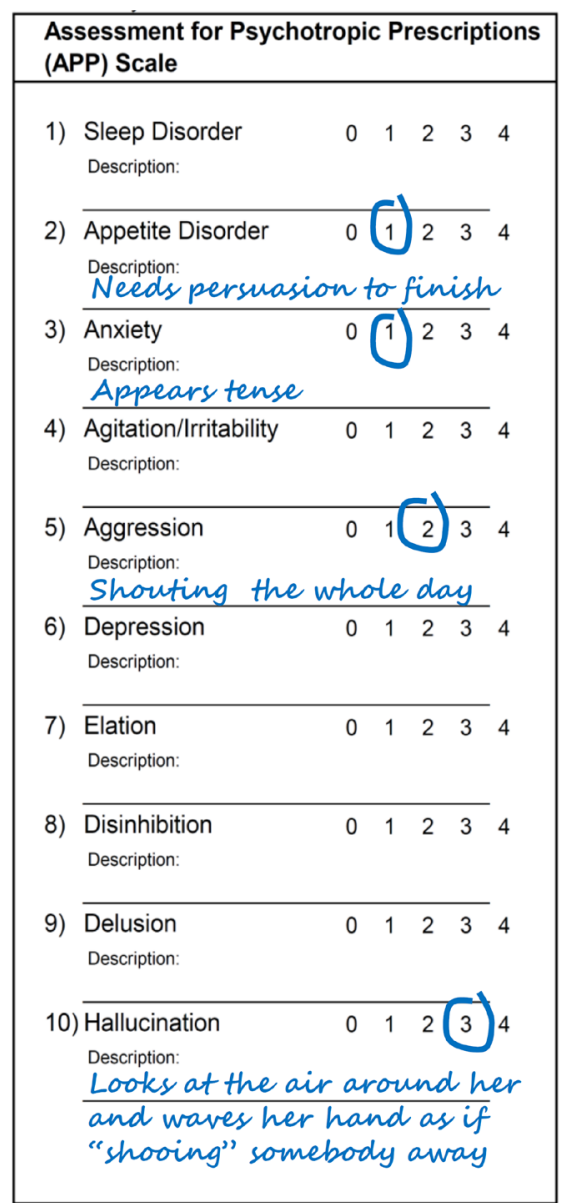

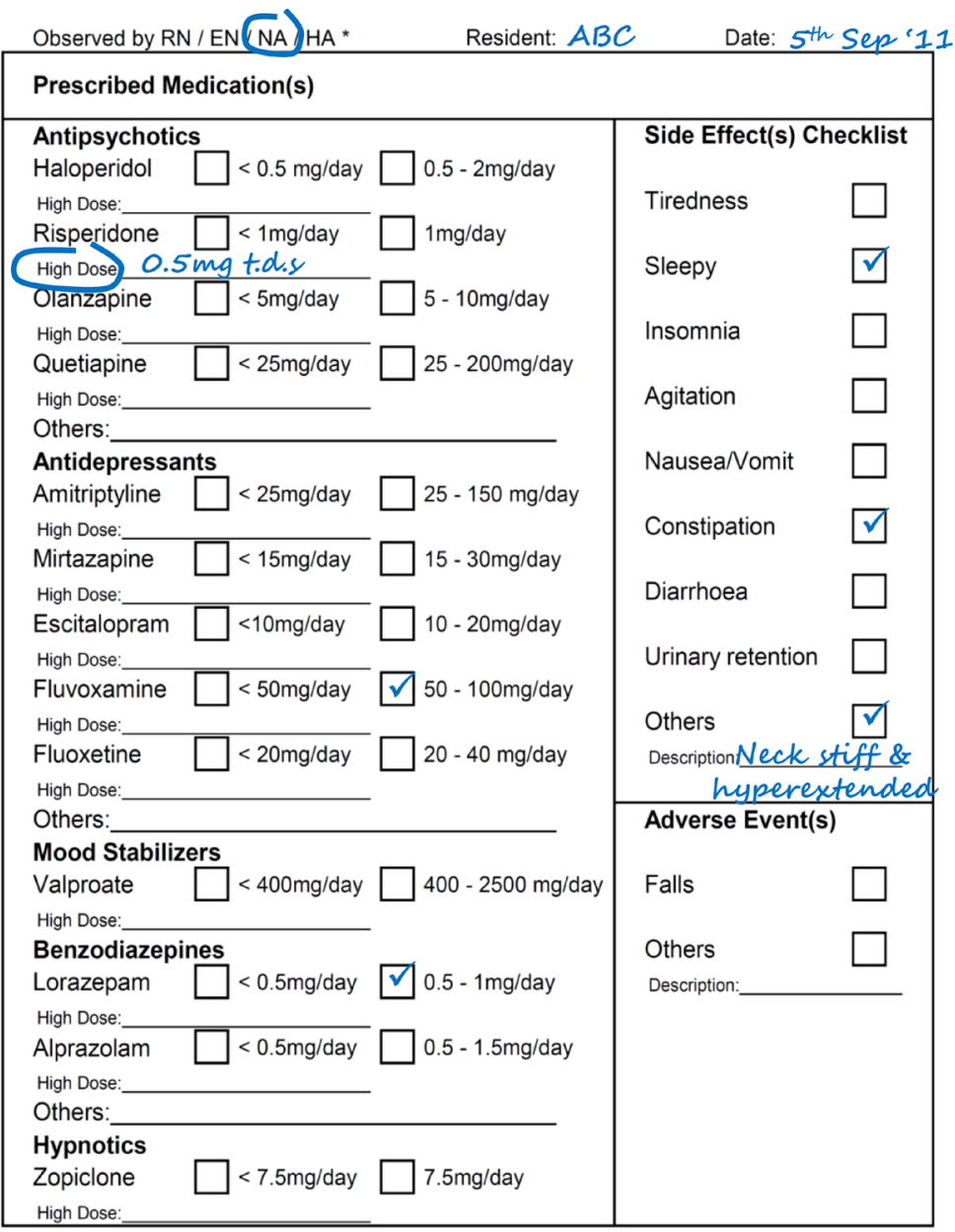

